\title{
Prophylactic and therapeutic efficacy of an attenuated Listeria monocytogenes-based vaccine delivering HPV16 E7 in a mouse model
}

\author{
YANYAN JIA，YUELAN YIN，FEIFEI DUAN，HONG FU，MAOZHI HU， \\ YUNFEI GAO, ZHIMING PAN and XINAN JIAO
}

Jiangsu Key Laboratory of Zoonosis, Yangzhou University, Yangzhou, Jiangsu 225009, P.R. China

Received May 25, 2012; Accepted August 2, 2012

DOI: $10.3892 /$ ijmm.2012.1136

\begin{abstract}
Listeria monocytogenes (L. monocytogenes) has been developed as a cancer vaccine vector due to its ability to elicit strong innate and adaptive immune responses. For clinical application, it is necessary to exploit a Listeria platform strain that is safe and that also retains its immunogenicity to develop vaccine candidates against cancer. In this study, a highly attenuated strain with a deletion of $a c t A / p l c B$ was employed as a vector to deliver the human papillomavirus type 16 (HPV16) E7 antigen, which was stably inserted into the chromosome of L. monocytogenes. The prophylactic and therapeutic efficacy of the recombinant $L$. monocytogenes strain expressing E7 (LM1-2-E7) were evaluated in C57BL/6 mice. In prophylactic tumor challenge assays, immunization with the recombinant strain LM1-2-E7 was able to protect against tumor formation in $87.5 \%$ of the mice, even after a second challenge, suggesting that this prophylactic immunization can provide long-lasting immunity. In the therapeutic setting, immunization with LM1-2-E7 led to tumor regression in $50 \%$ of the mice and suppressed tumor growth in the remaining mice. The results showed that the recombinant strain was cleared by the immune system within 5 days after immunization and induced a Th1 immune response against E7 peptide and E7-specific cytotoxic T-lymphocyte (CTL) killing activity without severe inflammatory responses in the spleen and liver. Markedly, recombinant Listeria strain resulted in preferential accumulation within tumor tissues and induced higher numbers of $\mathrm{CD}^{+} \mathrm{T}$ cells that infiltrated into the tumor, which were associated with retardation of tumor growth. Collectively, these data indicate that LM1-2-E7 is a possible vaccine candidate against cervical cancer.
\end{abstract}

Correspondence to: Professor Xinan Jiao, Jiangsu Key Laboratory of Zoonosis, Yangzhou University, 12 East Wenhui Road, Yangzhou, Jiangsu 225009, P.R. China

E-mail: xajiao@yahoo.com; jiao@yzu.edu.cn

Key words: attenuated Listeria monocytogenes, human papillomavirus type $16 \mathrm{E} 7$, cervical cancer, antitumor immunity

\section{Introduction}

Cervical cancer is the third most common cancer among women worldwide, and nearly half a million cases are diagnosed annually. In developing countries, it is the leading cause of cancer mortality in women $(1,2)$. Even with optimal treatment (primarily surgery, followed by chemotherapy and radiotherapy), $40 \%$ of cervical cancer patients succumb to this disease annually (3). Human papillomavirus type 16 (HPV16) is one of the principal pathogens in cervical cancer and is responsible for $>50 \%$ of cervical cancer cases $(3,4)$. The US Food and Drug Administration has approved 2 prophylactic HPV vaccines, Gardasil (Merck) and Cervarix (GlaxoSmithKline) to prevent cervical cancer and the prophylactic vaccines can significantly reduce the incidence of HPV-related cancers $(1,5)$. However, since the vaccine is based on the virion capsid proteins (L1, L2), which are absent in cervical cancer, they are unlikely to be effective in controlling pre-existing HPV infections or HPV-associated lesions (6). Therefore, it is necessary to develop both prophylactic and therapeutic vaccines against cervical cancer. The 2 major HPV oncoproteins, E6 and E7, which are consistently expressed in cervical cancer cells, are essential for the malignant transformation and maintenance of tumor cells (7). Cellular immunity to E7/E6 is associated with the clearance of premalignant HPV16 lesions, therefore, E6 and E7 are ideal targets for cervical cancer immunotherapy (8).

Listeria monocytogenes (LM) as a vaccine vector has been widely used to deliver tumor-associated antigens (TAAs) for cancer immunotherapy $(9,10)$. L. monocytogenes is a facultative intracellular bacterium that has the unusual ability to escape from the phagosome and multiply in the cytoplasm of cells (11). L. monocytogenes directly infects antigen-presenting cells (APCs), such as dendritic cells and macrophages, thereby delivering TAAs into their cytoplasm, resulting in processing and presentation of TAAs to the immune system, which induces antigen-specific $\mathrm{CD}^{+}$and $\mathrm{CD}^{+} \mathrm{T}$ cell responses $(12,13)$. Hence, recombinant $L$. monocytogenes-based vaccines expressing TAAs, including endoglin (CD105) (14), human prostate-specific antigen (PSA) (15), HER-2/neu (16) and HPV16 E7 $(17,18)$ have been developed for cancer immunotherapy in preclinical and clinical trials. Previously, Gunn et al 
(19) developed an LM-LLO-E7 vaccine, which carried a plasmid that contained an hly promoter to drive the expression of the LLO-E7 fusion protein in the presence of chloramphenicol. Verch et al reported that Listeria-based antibiotic resistance gene-free vaccine LMdd (pTV3) was constructed, but only attenuates the vector by $0.5-1 \log (20,21)$. However, for clinical application, the safety and ability of a live recombinant vaccine to stably express foreign antigens need to be assessed.

In the current study, an attenuated L. monocytogenes with deletions of $a c t A$ and $p l c B(\mathrm{LM} \triangle a c t A / p l c B$, in brief: LM1-2) was employed as a vaccine vector to deliver HPV16 E7. ActA is a major virulence protein that induces the rapid polymerization of filamentous actin and propels L. monocytogenes through the cytoplasm and into neighboring cells (22). Pamer (23) reported that ActA-deficient mutants are highly attenuated. Additionally, $\mathrm{PlcB}$ has been demonstrated to be important in second vacuolar escape (24). Previous studies analyzed a double mutant with $a c t A$ and $p l c B$ in adult volunteers and indicated that the mutant was a safe vector for clinical application $(25,26)$. Also, the E7 antigen was integrated into the chromosome of L. monocytogenes using the integration vector pIMK2-SPhly, which provides an effective tool to secretly express the heterologous proteins (27).

Results of the present study showed that the highly attenuated LM1-2-E7 strain induced E7-specific cell-mediated immunity and exhibited significant prophylactic and therapeutic efficacy against cervical cancer in a murine model. In addition, the antitumor efficacy was associated with intratumoral $\mathrm{CD}^{+} \mathrm{T}$ cell infiltration and preferential accumulation of LM1-2-E7 within tumor xenografts.

\section{Materials and methods}

Peptide, bacteria and plasmid. HPV16 E7 $7_{49-57}$ (RAHYNIVTF) peptide for the $\mathrm{H}-2 \mathrm{D}^{\mathrm{b}}$ restricted epitope was synthesized by Beijing Scilight Biotechnology, LLC. (Beijing, China). The plasmid pIMK2-SPhly was generously provided by Professor Chakraborty (Justus Liebig University, Giessen, Germany). Strain yzuLM4 (serotype 1/2a) was isolated and preserved in our laboratory. The LM1-2 strain was actA and $p l c B$ double mutant, which was previously constructed by homologous recombination (28).

Mice, cell lines and media. C57BL/6 mice (6-8 weeks old) were purchased from the Comparative Medical Center of Yangzhou University. Animals were housed and used in accordance with the protocols approved by the institutional animal experimental committee. The TC- 1 cell line was purchased from Beijing Hualisentai Bio-Scientific, Co., Ltd. (Beijing, China). TC-1 cells are C57BL/6 lung tumor epithelial cells immortalized with HPV16 E6/E7 and transformed with the c-Ha-ras oncogene. Cells were cultured in RPMI-1640, supplemented with $10 \%$ fetal calf serum (FCS), $1 \mathrm{mmol} / 1$ sodium pyruvate, $100 \mathrm{U} / \mathrm{ml}$ penicillin and $100 \mu \mathrm{g} / \mathrm{ml}$ streptomycin in a $37^{\circ} \mathrm{C}$ incubator with $5 \% \mathrm{CO}_{2}$.

Construction of the recombinant LM1-2-E7 strain. The E7 fragment was amplified and cloned into vector pIMK2-SPhly using primers $\mathrm{E} 7$ forward, (GACGGATCCCATGGAGATAC ACCTAC) and E7 reverse (CCGCTCGAGTTATGGTTTCTG
AGAACA) with restriction enzyme sites BamHI and XhoI. The resulting plasmid pIMK2-SPhly-E7 and control plasmid pIMK2-SPhly were electroporated into competent LM1-2, which were designed as the recombinant $L$. monocytogenes strain LM1-2-E7 and LM1-2-control, respectively.

Western blotting. LM1-2-E7 and LM1-2-control were cultured overnight in brain heart infusion (BHI) broth at $37^{\circ} \mathrm{C}$ and their supernatants were collected by centrifugation at $8,000 \mathrm{rpm}$ for $10 \mathrm{~min}$. Secreted proteins in the culture supernatants were precipitated with trichloroacetic acid (TCA; Sigma-Aldrich, St. Louis, MO, USA) and resuspended in $1.5 \mathrm{mM}$ Tris- $\mathrm{HCl}$ buffer. Then, the precipitates were lysed by ultrasonic waves. The secreted proteins and lysates were separated by $4-12 \%$ SDS-PAGE, transferred to a nitrocellulose membrane and detected using an anti-E7 monoclonal antibody (Clone 8C9; Invitrogen Life Technologies, Carlsbad, CA, USA). The signals were measured using ECL detection reagents (Thermo Scientific, Rockford, IL, USA) and exposure to Hyperfilm.

In vitro stability assay. The stability of LM1-2-E7 was determined by serially passaging for 40 times without antibiotics and subsequently analyzing the expression of E7 in the 20th, 30th and 40th passages.

Immunization of mice with LM1-2-E7. C57BL/6 mice (6-8 weeks old) were intraperitoneally immunized with LM1-2-E7 $\left(0.1 \mathrm{LD}_{50}, 5 \times 10^{7} \mathrm{CFU}\right)$ or LM1-2-control $\left(0.1 \mathrm{LD}_{50}\right.$, $5 \times 10^{7} \mathrm{CFU}$ ) or phosphate-buffered saline (PBS) buffer on days 0 and 7 . Seven days after the booster immunization, mice were used for ELISPOT assay, cytotoxicity assay in vivo and histopathological study. The groups treated with PBS buffer or LM1-2-control were used as negative controls.

Enzyme-linked immunosorbent spot (ELISPOT) assay. The ELISPOT assay was performed as previously described (29). The spleens were harvested and processed into single cell suspensions. The cell suspensions were treated with ammonium chloride (ACK) buffer to lyse the erythrocytes and washed twice with complete RPMI-1640 medium. A total of $5 \times 10^{5}$ cells/well was incubated in anti-murine interferon (IFN)- $\gamma /$ interleukin (IL)-4-coated ELISPOT plates. The cells were stimulated in triplicate in C-RPMI medium as a negative control and pulsed with $2 \mu \mathrm{M} \mathrm{E7}_{49-57}$ (RAHYNIVTF) peptide or with concanavalin A (ConA, $5 \mu \mathrm{g} / \mathrm{ml}$; Sigma, St. Louis, MO, USA) as a positive control. After $48 \mathrm{~h}$ of incubation at $37^{\circ} \mathrm{C}$, the plates were developed according to the manufacturer's protocol (BD Pharmingen, San Diego, CA, USA). The monoclonal antibodies that were used for the ELISPOT assay were R4-6A2 for IFN- $\gamma$, XMG1.2 for biotinylated IFN- $\gamma$, BVD4-1D11 for IL-4 and BVD6-24G2 for biotinylated IL-4 (BD Pharmingen). The spots were counted using an automated ELISPOT Bioreader 5000 (ImmunoBioSystem, The Colony, TX, USA).

Cytotoxicity assay. To determine specific cytotoxicity in vivo, the splenocytes were pooled from naive C57BL/6 mice and divided into 2 groups: the cell suspension for 1 group was incubated with the E7 peptide at $37^{\circ} \mathrm{C}$ for $45 \mathrm{~min}$ and subsequently labeled with $2.5 \mu \mathrm{M}$ Carboxyfluorescein succinimidyl ester (CFSE ${ }^{\text {high }}$; Molecular Probes, Invitrogen Life Technologies) 


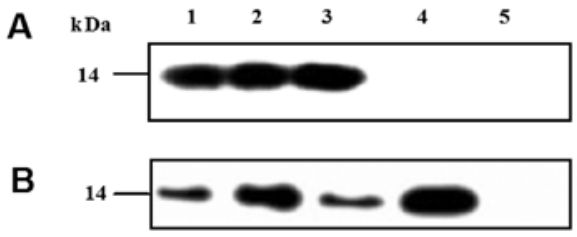

Figure 1. Western blot analysis of LM1-2-E7. (A) Western blot analysis of E7 expression in the LM1-2-E7 strain. Both the secreted proteins and lysates were detected using an anti-E7 monoclonal antibody. Lanes 1-2, the lysates and secretory proteins from LM1-2-E7, respectively; lane 3, HPV16 E7 protein expressed in E. coli as a positive control; lanes 4-5: the lysates and secretory proteins from LM1-2-control. (B) Stability assay in LM1-2-E7 strain after 40 generations. Lanes 1-3: secreted proteins from the 20th, 30th and 40th passages of LM1-2-E7; lane 4, HPV16 E7 protein expressed in $E$. coli as a positive control; lane 5, LM1-2-control as a negative control.

buffer at $37^{\circ} \mathrm{C}$ for $10 \mathrm{~min}$, whereas another group was incubated without the peptide and labeled with $\mathrm{CFSE}^{\text {low }}(0.25 \mu \mathrm{M})$ buffer. CFSE ${ }^{\text {high }}$ and CFSE ${ }^{\text {low }}$ cells were mixed in a 1:1 ratio and $10^{7}$ cells were intravenously injected into immunized mice. Twenty-four hours later, the spleens of the immunized mice were processed into single cell suspensions and analyzed by flow cytometry (FACScan; Becton Dickinson) to determine the ratio of $\mathrm{CFSE}^{\text {high }}$ to $\mathrm{CFSE}^{\text {low }}$ cells. The percentage of specific lysis was calculated using the following formula: percent specific lysis=100-[100x (\% CFSE ${ }^{\text {high }}$ immunized/\% CFSE $^{\text {low }}$ immunized $) /\left(\% \mathrm{CFSE}^{\text {high }}\right.$ control $/ \% \mathrm{CFSE}^{\text {low }}$ control)] (30).

Histopathological study. The sections of the spleens and livers were fixed in $13 \%$ neutral buffered formalin. Paraffinembedded sections were cut at $5 \mu \mathrm{m}$, stained with hematoxylin and eosin (H\&E), and examined for histological lesions under a microscope (Leica Microsystems, Wetzlar, Germany).

Prophylactic and therapeutic tumor load experiments. In a prophylactic experiment, C57BL/6 mice (6-8 weeks old, 8 per group) were intraperitoneally immunized with LM1-2-E7 or LM1-2-control or PBS buffer on days 0 and 7 and subcutaneously challenged on day 11 with $2 \times 10^{5}$ TC- 1 cells. The mice were monitored for tumor formation.

In a therapeutic setting, C57BL/6 mice (8 per group) were subcutaneously injected with $2 \times 10^{5} \mathrm{TC}-1$ cells on the left flank. When the tumor size reached an average diameter of $5 \mathrm{~mm}$ on day 7 after tumor cell inoculation, the mice received LM1-2-E7 or LM1-2-control or PBS buffer intraperitoneally on days 7 and 14 . The tumors were monitored every 3 days with calipers and the longest and shortest surface diameters were recorded for each individual tumor. Tumor volume was calculated as the following: length $\mathrm{x}$ (width) $)^{2} / 2$ (31).

Analysis of $C D 8^{+} T$ cells in the tumor. The tumors in the immunized groups were excised on day 7 after the second immunization, minced using a sterile razor blade and digested with a buffer containing $2 \mathrm{mg} / \mathrm{ml}$ collagenase type I and $12 \mathrm{U} / \mathrm{ml}$ DNase in PBS buffer. After a $2 \mathrm{~h}$ incubation at $37^{\circ} \mathrm{C}$ with agitation, single cell suspensions were harvested after filtration through a nylon mesh, stained with anti-CD3-FITC (clone145-2C11; BD Pharmingen) and anti-CD8-APC monoclonal antibodies (clone53-6.7; BD Pharmingen) and analyzed by FACS.

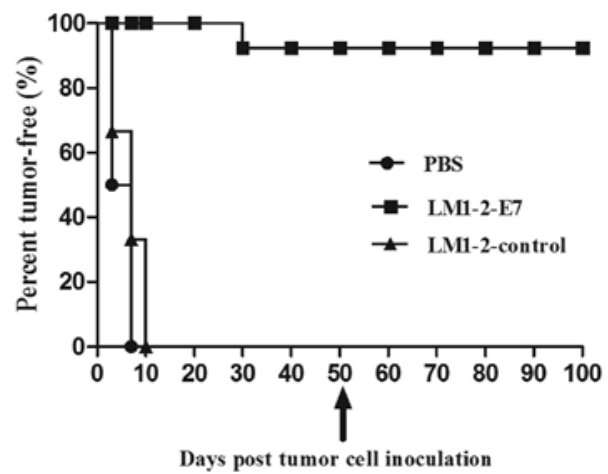

Figure 2. Preventive effect of immunization with LM1-2-E7. Three groups of 8 mice were intraperitoneally immunized with LM1-2-E7, LM1-2-control and PBS buffer (twice at 1-week intervals), respectively, and challenged with a subcutaneous injection of $2 \times 10^{5} \mathrm{TC}-1$ cells on the left flank 4 days following the second vaccination. Mice were observed for tumor development. Seven tumor-free mice were rechallenged with TC-1 cells on Day 50 after the first challenge (arrow) and were observed for tumor formation. The experiment was repeated twice showing similar results.

For immunohistochemistry analysis, tumor specimens in the immunized groups were fixed in 13\% neutral buffered formalin. Paraffin-embedded sections were cut at $5 \mu \mathrm{m}$ and incubated with anti-mCD8 (clone53-6.7; R\&D Systems, Minneapolis, MN, USA) overnight at $4^{\circ} \mathrm{C}$. The anti-mCD8 antibody was visualized through HRP-DAB Cell and Tissue Staining kit (R\&D Systems) and counterstained with H\&E.

Bacterial translocation studies. Tumor-bearing mice (7 days after the tumor cell inoculation) received a single intraperitoneal immunization (0.1 LD LD $_{50}$ LM1-2-E7). The spleens, livers and tumors of 3 mice were homogenized on days 1,2, 3 and 5 post-immunization. The bacterial numbers were determined by plating the cell suspensions on BHI agar. The tumor tissues at 1 day post-immunization were cut into smaller sections, fixed in a solution of $2.5 \%(\mathrm{w} / \mathrm{v})$ glutaraldehyde in $0.1 \mathrm{M}$ cacodylate buffer, dehydrated and embedded in Epon. Ultrathin sections were cut and stained with uranyl acetate and Reynold's lead citrate. The sections were examined using a Transmission Electron Microscope (TEM; JEOL Ltd., Tokyo, Japan).

Statistical analysis. Statistical analyses for in vitro and in vivo experiments were carried out using the GraphPad Software package (GraphPad Software, La Jolla, CA, USA). Student's t-test and one-way ANOVA were used for analysis of the comparisons between the groups. Statistical significance was set at ${ }^{*} \mathrm{P}<0.05,{ }^{* *} \mathrm{P}<0.01$ and ${ }^{* * *} \mathrm{P}<0.001$.

\section{Results}

Construction of LM1-2-E7 to express and secrete the HPV16 E7 protein. The recombinant attenuated LM1-2-E7 strain, which integrated the encoding gene of the HPV16 E7 into the chromosome of the LM1-2 strain using pIMK2-SPhly, was constructed. Western blotting revealed that the recombinant strain LM1-2-E7 expressed and secreted the HPV16 E7 protein (Fig. 1A). Moreover, E7 was stably expressed for at least 40 passages indicated by in vitro stability assay (Fig. 1B). 


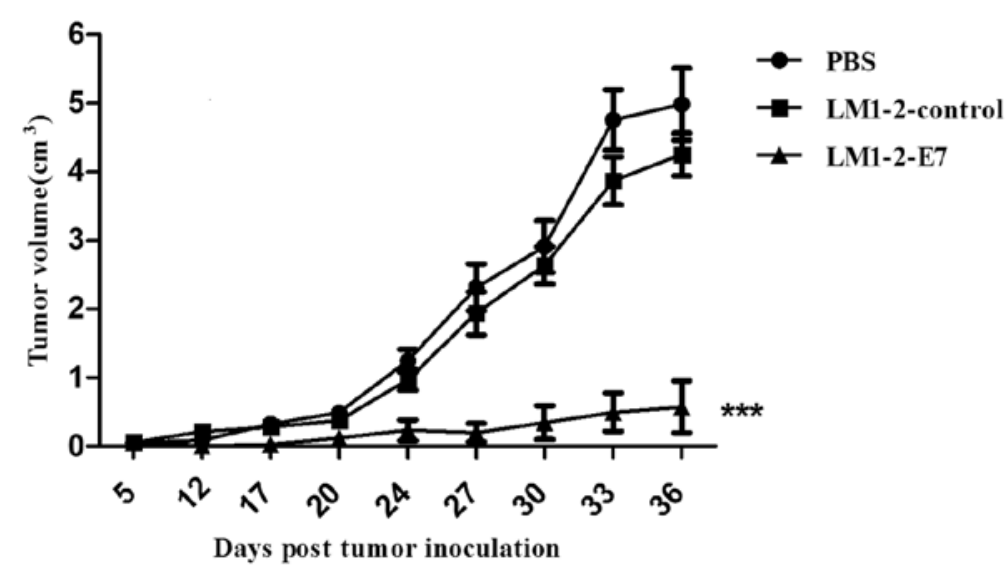

\begin{tabular}{|c|c|c|}
\hline \multirow[b]{2}{*}{ Day } & \multicolumn{2}{|c|}{ Tumor-fre } \\
\hline & 7 & 36 \\
\hline LM1-2-E7 & $8: 8$ & $4: 8$ \\
\hline LM1-2-control & $8: 8$ & $0: 8$ \\
\hline \multirow[t]{2}{*}{ PBS } & $8: 8$ & $0: 8$ \\
\hline & \multicolumn{2}{|c|}{ Survival } \\
\hline Day & 7 & 36 \\
\hline LM1-2-E7 & $8: 8$ & $8: 8$ \\
\hline LM1-2-control & $8: 8$ & $7: 8$ \\
\hline PBS & $8: 8$ & $3: 8$ \\
\hline
\end{tabular}

Figure 3. Therapeutic effect of vaccination with LM1-2-E7. Three groups of 8 mice were subcutaneously injected with $2 \times 10^{5}$ TC-1 cells on the left flank on day 0. The mice were administered LM1-2-E7 or LM1-2-control or PBS buffer on days 7 and 14. Tumors were monitored twice weekly and tumor size was measured until day 36 when the majority of the mice in the PBS group died. The table shows the number of mice in each group which were either tumor-free (upper panel) or survived (lower panel), compared to the total number of mice/group on day 7 or 36 post-tumor cell inoculation. Data were expressed as the means \pm SD values from 3 independent experiments. ${ }^{* * *} \mathrm{P}<0.001$ vs. the LM1-2-control group and the PBS group.

Vaccination with LM1-2-E7 protects mice against tumor cell challenge. The preventive effect of the LM1-2-E7 vaccine was assessed in the TC-1 tumor model. Mice were challenged with TC-1 cells on day 4 after the second immunization and were observed for tumor development. In the LM1-2-E7vaccinated group, 7 mice were tumor-free until day 50 , excluding 1 mouse $(87.5 \%)$ that indicated tumor growth on day 32 after the tumor cell challenge. By contrast, tumors appeared in all of the mice on day 7 in the PBS group and on day 10 in the LM1-2-control group (Fig. 2). The results demonstrate that the LM1-2-E7 strain confers significant preventive efficacy. Furthermore, 7 tumor-free mice were rechallenged with TC-1 cells on day 50 and all of the mice rejected the tumor challenge 50 days after the second tumor cell injection. The tumor-free mice appeared to be healthy and presented no weight loss by the end of the study. This finding indicates that the mice which are vaccinated with LM1-2-E7 have long-lasting protection and elicit a memory response against HPV16.

LM1-2-E7 strain causes regression of established tumors in mice. To examine the therapeutic efficacy of the LM1-2-E7 vaccine candidate, C57BL/6 mice were subcutaneously injected TC-1 cells and intraperitoneally immunized with LM1-2-E7, LM1-2-control, or PBS buffer on days 7 and 14 and tumor growth were measured. As shown in Fig. 3, the tumor volumes in the mice that were immunized with LM1-2-E7 were significantly reduced compared to those in the LM1-2-control or the PBS group $(\mathrm{P}<0.001)$. Moreover, 4 of the 8 mice in the LM1-2-E7-immunized group remained tumor-free. Additionally, the mice immunized with LM1-2-E7 survived tumor invasion on day 36. By contrast, 7 mice survived tumor invasion in the LM1-2-control group and only 3 mice in the PBS group. These results indicate that the LM1-2-E7 strain exhibits therapeutic activity in the TC-1 mouse tumor model.

LM1-2-E7 strain elicits E7-specific cellular immune responses in mice. In order to measure the cellular immune responses elicited by LM1-2-E7, the splenocytes were isolated on Day 7 from the second immunization, and the number of IFN- $\gamma /$ IL-4-secreting cells after stimulation with E7 $49-57$ peptide was analyzed using an ELISPOT assay. The mean number of IFN- $\gamma$-secreting cells in the LM1-2-E7-immunized group was significantly higher than the number of IL-4secreting cells $(\mathrm{P}<0.01)$ and significantly increased compared to those of the control groups $(\mathrm{P}<0.01)$. These results indicate that mice immunized with LM1-2-E7 developed cellular immune responses against the E7 peptide (Fig. 4).

Cytotoxic T-lymphocytes (CTLs) are a critical component of the immune response to tumors. Thus, the E7-specific CTL responses in vivo were determined. The results indicate that LM1-2-E7-vaccinated mice exhibited the highest cytolytic activity $(53.49 \pm 3.63 \%)$ against E7 $7_{49-57}$-loaded target cells in comparison with the cytolytic activity $(13.96 \pm 2.05 \%)$ found in the immunized with LM1-2-control and $(2.0 \pm 0.76 \%)$ in the PBS group.

Increased frequency of tumor-infiltrating $C D 8^{+} T$ cells in tumors. Tumor-infiltrating lymphocytes (TILs) are a part of the tumor surveillance system. The $\mathrm{CD}^{+} \mathrm{T}$ cells were analyzed in the tumors from all the groups. A higher number of tumor-infiltrating $\mathrm{CD}^{+} \mathrm{T}$ cells $(22.45 \pm 2.66 \%)$ was detected in the LM1-2-E7 group compared to the controls $(17.45 \pm 2.1 \%$, LM1-2-control; $8.55 \pm 0.96 \%$, PBS group) (Fig. 5A and B). A similar result was also found in the immunohistochemistry of the excised tumor tissues (Fig. 5C). These results indicate that the LM1-2-E7 vaccine could enhance antitumor immunity by increasing the infiltration of $\mathrm{CD}^{+} \mathrm{T}$ cells into the tumor.

Histopathological study of the organs of the immunized mice. The spleens from the LM1-2-E7-immunized group and LM1-2-control group did not reveal any significant pathological lesions and the liver sections revealed inflammatory cell infiltration in the hepatic lobules. The results demonstrate that LM1-2-E7 and LM1-2-control induce a mild inflammatory response with no necrosis or structure damage (Fig. 6). 


\section{A}
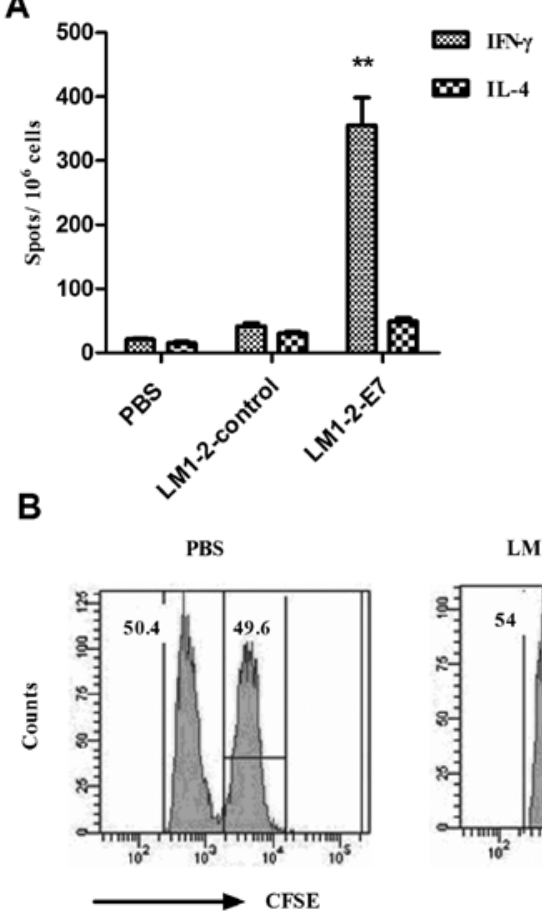

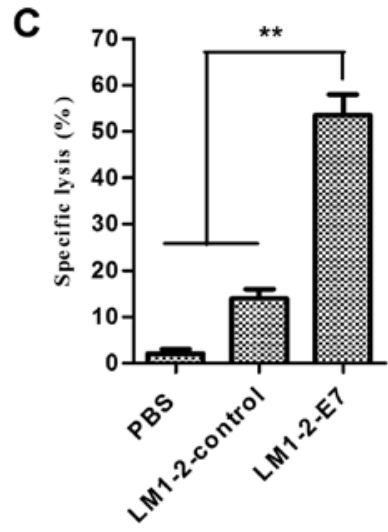

LM1-2-control

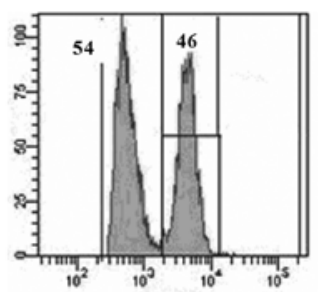

LM1-2-E7

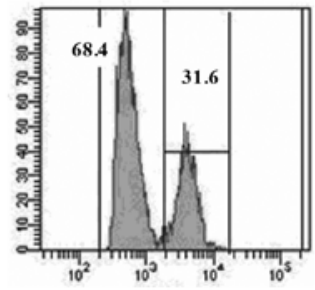

Figure 4. Cellular immune responses elicited by LM1-2-E7. (A) Cytokine (IFN- $\gamma /$ IL-4) secretion after intraperitoneal immunization with LM1-2-E7. On day 7 following the second immunization, the splenocytes of the immunized mice (5 mice/group) were isolated, pulsed with the E7 peptide in vitro and in the presence of ConA as a positive control. After $48 \mathrm{~h}$ of incubation, the plates were developed according to the manufacturer's protocol to detect the number of cytokine (IFN- $\gamma / \mathrm{IL}-4)$ secreting cells. Data are expressed as the means \pm SD values from 3 independent experiments. ${ }^{* *} \mathrm{P}<0.01$ vs. the LM1-2-control and the PBS group. (B) Cytotoxic activity in vivo. A mixture of CFSE-labeled syngeneic splenocytes that were pulsed with the H-2D ${ }^{\mathrm{b}}$ E7 peptide (CFSE ${ }^{\text {high }}$ ) or not pulsed with the peptide (CFSE ${ }^{\text {low }}$ ) was intravenously injected into the immunized and control mice on day 7 after the second immunization (5 mice/group). After $24 \mathrm{~h}$, the mice were sacrificed and the specific killing of E7 ${ }_{49-57}$-positive targets were analyzed using FACS. The representative samples of in vivo killing of E7 ${ }_{49-57}$-positive targets are shown. (C) Cytolytic activity in different groups in immunized mice. Data were expressed as the means \pm SD values from 3 independent experiments. ${ }^{* *} \mathrm{P}<0.01$ vs. the LM1-2-control group and PBS group.

A

PBS
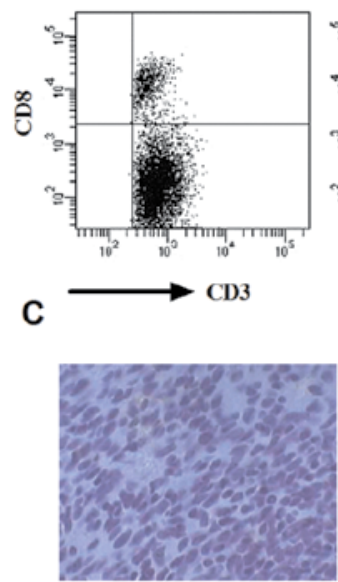

LM1-2-control
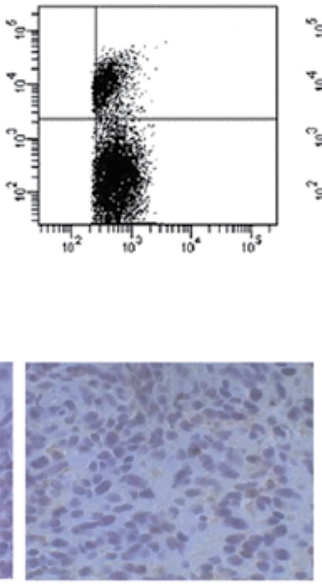

LM1-2-E7
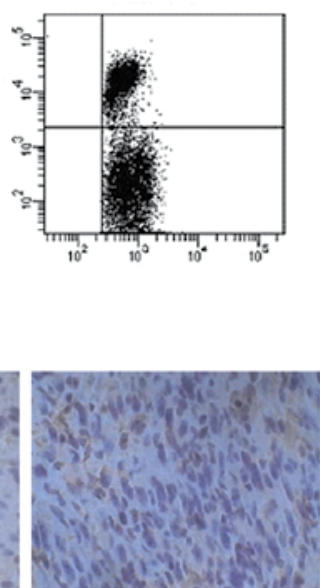

B

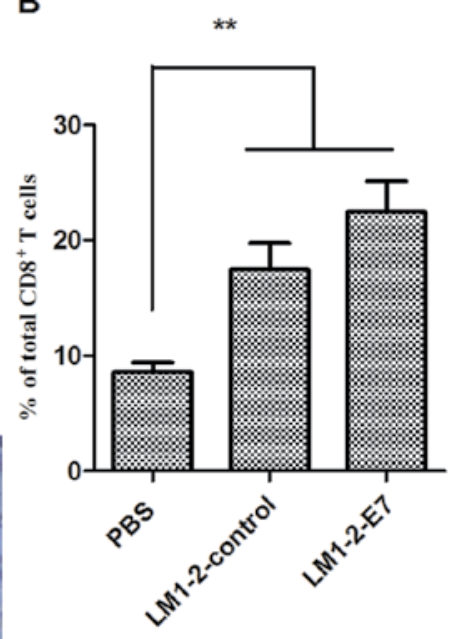

Figure 5. Analysis of tumor infiltrating $\mathrm{CD}^{+} \mathrm{T}$ cells. (A) The tumors were excised on day 7 after the second immunization (5 mice/group) and single cell suspensions from the tumors were stained with anti-CD3-FITC and anti-CD8-APC monoclonal antibodies and analyzed using FACS. (B) The number of tumor infiltrating $\mathrm{CD}^{+} \mathrm{T}$ cells in different groups. (C) $\mathrm{CD} 8^{+} \mathrm{T}$ cells of the tumor samples were analyzed for immunohistochemistry with magnification $\mathrm{x} 200$. Images are representative of 9 mice in the immunized groups and the PBS group. Data were expressed as the means \pm SD values from 3 independent experiments. ${ }^{* * *} \mathrm{P}<0.01$ vs. the PBS group.

Distribution of the LM1-2-E7 strain after a single intraperitoneal immunization. Tumor-bearing mice were intraperitoneally immunized with a single dose of LM1-2-E7 and the number of viable bacteria was determined in the spleen, liver and tumor homogenates. As shown in Fig. 7A, the LM1-2-E7 bacteria were rapidly cleared from the spleen and 
PBS

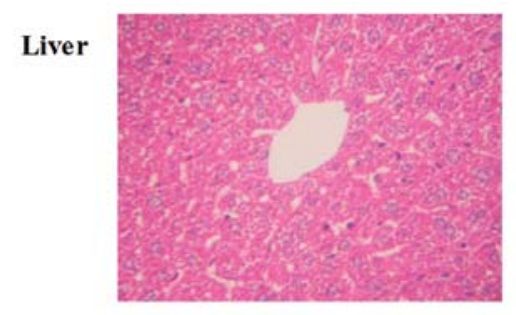

Spleen

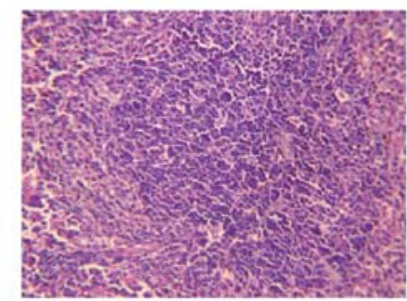

LM1-2-control
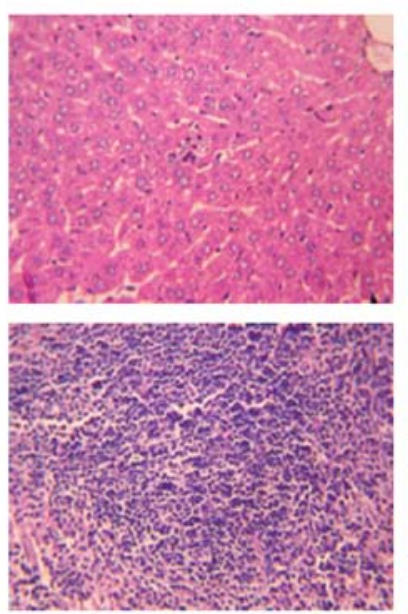

LM1-2-E7
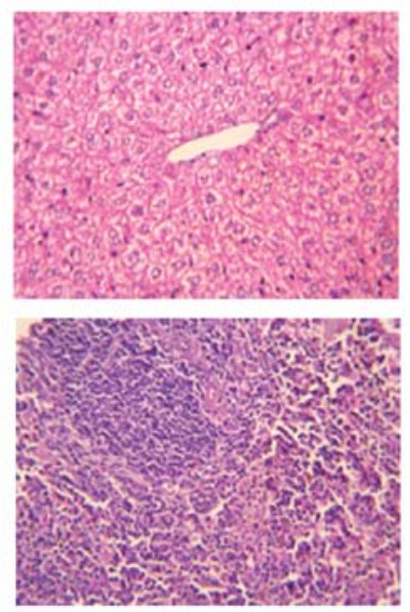

Figure 6. Histopathological studies of the organs of the immunized mice. On day 7 after the second immunization, the spleens and livers (5 mice/group) were harvested and stained by H\&E. Images are representative of 5 mice in the immunized group and the control groups (original magnification, $\mathrm{x} 400$ ).
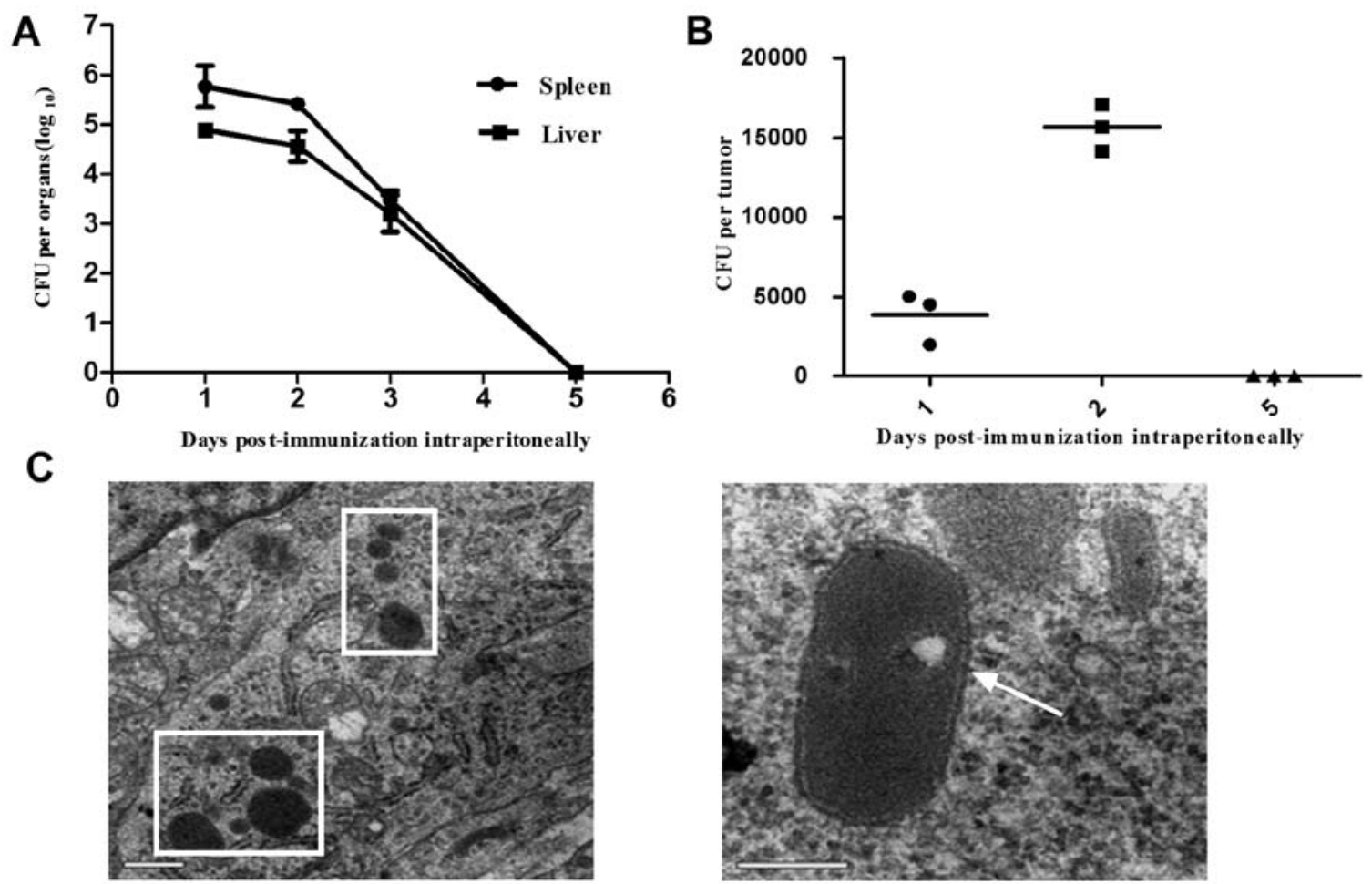

Figure 7. LM1-2-E7 translocation study. C57BL/6 mice were subcutaneously injected with TC-1 cells on day 0 and intraperitoneally immunized with a single dose of $0.1 \mathrm{LD}_{50} \mathrm{LM1}-2-\mathrm{E} 7$ on day 7. The spleens, livers and tumors of 3 mice were homogenized on days 1, 2, 3 and 5 post-immunization. The bacterial numbers were determined by plating the cell suspension on BHI agar. (A) The kinetics of LM1-2-E7 CFU in the spleens and livers. The y-axis represents the logarithm of viable bacterial CFU to base 10 in the spleens and livers. (B) The data show the kinetics of CFU in the tumors. (C) A TEM analysis indicates that the LM1-2-E7 infected tumors with high efficacy after a single intraperitoneal immunization. Bar, $500 \mathrm{~nm}$ (left) and $200 \mathrm{~nm}$ (right). White arrows, LM1-2-E7 bacteria. Data were expressed as the means \pm SD values from 2 independent experiments.

liver without damaging their structures. On day 1 post-immunization, LM1-2-E7 bacteria were detected in tumor tissue and the number of bacteria peaked on day 2. TEM demonstrated that the LM1-2-E7 strain infected the tumors in vivo with a high efficacy (Fig. 7C). On day 5 post-immunization, LM1-2-E7 disappeared from the spleens, livers and tumors of the mice (Fig. 7A and B).

\section{Discussion}

There are currently prophylactic vaccines against HPV for clinical use, however, the cost of these vaccines is prohibitive in developing countries (1). In addition, these prophylactic vaccines do not generate therapeutic effects (32). The prophylactic and therapeutic $L$. monocytogenes-based vaccine 
(LM1-2-E7) was developed in the present study. LM1-2-E7 was able to protect $87.5 \%$ of the mice even 50 days after the second tumor cell challenge. Also, immunization with the LM1-2-E7 strain was able to induce tumor regression in 50\% of the mice in the therapeutic experiment.

To avoid serious toxicity of $L$. monocytogenes, a highly attenuated strain $\mathrm{LM} \triangle a c t A / p l c B$ was selected as a vaccine vector. Due to deletion of the virulence factors $a c t A$ and $p l c B$, the pathogenicity of $\mathrm{LM} \triangle a c t A / p l c B$ is significantly decreased, the $\mathrm{LD}_{50}$ is $3 \log$ s higher than that of the parent strain yzuLM4 (data not shown). As indicated in tissue sections, only lymphocyte infiltration was observed in the livers from LM1-2-E7-immunized mice. Also, the results demonstrated that the attenuated LM1-2-E7 strain was cleared by the immune system within 5 days post-immunization, which is in line with the $\mathrm{LM} \triangle a c t A / p l c B$ strain (33). Although virulence of the LM1-2-E7 strain is reduced, LM1-2-E7 retains the ability to induce strong E7-specific immune responses.

In addition to choosing the attenuated strain as backbone, an integration vector pIMK2-SPhly was used for the construction of recombinant strain. The E7 antigen was stably integrated into the Listeria chromosome via pIMK2-SPhly and secretly expressed by recombinant strain, which is the basis of the E7 antigen entered into the MHC class I pathway (27). Moreover, stability assay in vitro suggests that the E7 antigen was stably expressed for at least 40 passages, which is appropriate for clinical immunization.

Antigen-specific $\mathrm{T}$ cell responses are most critical for the regression of established tumors and protection against tumor challenge. IFN- $\gamma$ and IL- 4 secreted by Th1 cells and Th 2 cells in LM1-2-E7 immunized mice were determined by ELISPOT assay. The increased secretion of the Th1 cytokine IFN- $\gamma$ indicated that the immune response elicited by LM1-2-E7 was biased toward the Th1 type against the $\mathrm{E}_{49-57}$ peptide. These Th1 T cells have a pivotal role in antitumor immunity and contribute to APC maturation and the release of cytokines during $\mathrm{CD} 8^{+} \mathrm{T}$ cell proliferation and differentiation (34). Of note, our cytotoxicity activity in vivo revealed that the stimulated T cells in LM1-2-E7 immunized mice could destroy the target cells pulsed with the E7 peptide, which indicated that LM1-2-E7 could induce strong E7-specific CTL response in vivo.

The generation of $\mathrm{CD}^{+}$tumor-infiltrating lymphocytes (TILs) can be used as a surrogate marker for cancer vaccine efficacy and activated $\mathrm{CD} 8^{+} \mathrm{T}$ cells release cytolytic agents that attack and kill tumor cells (35). The number of tumorinfiltrating $\mathrm{CD}^{+} \mathrm{T}$ cells increased in the LM1-2-E7 group compared to the controls through the FACS and immunohistochemistry analyses, which was strongly associated with the better therapeutic efficacy in the LM1-2-E7-immunized mice. The higher number of $\mathrm{CD} 8^{+} \mathrm{T}$ cells may be depended on the cytosolic location of L. monocytogenes.

Our results show that the recombinant Listeria strain could infect and reside in the tumors, so E7 antigen delivered by the recombinant Listeria strain is targeted to the tumor tissue. Yu et al (36) suggested that the survival and replication of L. monocytogenes in tumors is dependent on the tumor immune microenvironment and vascularization, which provides a sanctuary for bacteria to escape clearance by the immune system. L. monocytogenes in tumors may directly lyse tumor cells or stimulate homing effector cells by releasing cytokines and chemokines to destroy tumor cells. Gravekamp and Paterson also reported that L. monocytogenes can efficiently kill tumor cells through a dual mode of action, which involves both direct kill and CTL responses to the Listeria antigen (37).

In the present study, L. monocytogenes exerts a strong adjuvant effect, which is associated with a strong innate immunity induced by L. monocytogenes (12). One manifestation is that 2 -fold increase of tumor-infiltrating $\mathrm{CD} 8^{+} \mathrm{T}$ cells caused in the LM1-2-control group compared to the PBS group. In vivo tumor regression assay showed that LM1-2control can prolong the survival time of tumor-bearing mice, which mainly produced an effect by L. monocytogenes.

The TC-1 cell line which expresses HPV E6 and E7 oncoproteins was applied to establish an HPV16-positive cancer-associated tumor model. The TC-1 tumor model in C57BL/6 mice is a widely-used model for use in vaccine research against cervical cancer $(38,39)$. Thus, the antitumor efficacy of LM1-2-E7 was initially evaluated in the TC-1 tumor model. However, this transplantable tumor model has several limitations, such as the inability to accurately mimic the immunoinhibitory effects of the tumor microenvironment (40). Hence, other cervical cancer models (a transgenic mouse model) will be selected to evaluate the potency of this vaccine in future studies.

In conclusion, our results demonstrate that LM1-2 is a possible vaccine vector, which is capable of delivering TAAs for cancer immunotherapy. LM1-2-E7 exerts a prophylactic effect on tumor growth and leads to the regression of established tumors expressing E7 antigen. The antitumor efficacy was associated with E7-specific CTL response and robust cellular immune responses elicited by recombinant strain. The results may be of importance in further investigations of this vaccine to combat cervical cancer and other HPV-associated cancers.

\section{Acknowledgements}

The authors thank Professor T. Chakraborty for providing plasmid pIMK2-SPhly. This study was supported by grants from the National Basic Research Program of China (2012CB518805), the Science and Technology Support Program of Jiangsu Province (BE2012367), the Priority Academic Program Development of Jiangsu Higher Education Institutions (PAPD), the National Natural Science Foundation of China (no. 31101841) and the Provincial Government of Jiangsu, China (nos. BK2011446 and 2009KJA230001).

\section{References}

1. Cohen J: Public health. High hopes and dilemmas for a cervical cancer vaccine. Science 308: 618-621, 2005.

2. Jemal A, Bray F, Center MM, Ferlay J, Ward E and Forman D: Global cancer statistics. CA Cancer J Clin 61: 69-90, 2011.

3. Gatta G, Lasota MB and Verdecchia A: Survival of European women with gynaecological tumours, during the period 1978-1989. EUROCARE Working Group. Eur J Cancer 34: 2218-2225, 1998.

4. Schäfer A, Friedmann W, Mielke M, Schwartländer B and Koch MA: The increased frequency of cervical dysplasianeoplasia in women infected with the human immunodeficiency virus is related to the degree of immunosuppression. Am J Obstet Gynecol 164: 593-599, 1991. 
5. Haupt RM and Sattler C: HPV vaccine continues to be safe and effective, and its benefits continue to outweigh its risks. Expert Rev Vaccines 9: 697-701, 2010.

6. Wu TC: Therapeutic human papillomavirus DNA vaccination strategies to control cervical cancer. Eur J Immunol 37: 310-314, 2007.

7. zur Hausen H: Papillomaviruses and cancer: from basic studies to clinical application. Nat Rev Cancer 2: 342-350, 2002.

8. Hung CF, Wu TC, Monie A and Roden R: Antigen-specific immunotherapy of cervical and ovarian cancer. Immunol Rev 222: 43-69, 2008.

9. Shahabi V, Seavey MM, Maciag PC, Rivera S and Wallecha A: Development of a live and highly attenuated Listeria monocytogenes-based vaccine for the treatment of Her2/neu-overexpressing cancers in human. Cancer Gene Ther 18: 53-62, 2011.

10. Tangney $\mathrm{M}$ and Gahan CG: Listeria monocytogenes as a vector for anti-cancer therapies. Curr Gene Ther 10: 46-55, 2010.

11. Bruhn KW, Craft N and Miller JF: Listeria as a vaccine vector. Microbes Infect 9: 1226-1235, 2007.

12. Zenewicz LA and Shen H: Innate and adaptive immune responses to Listeria monocytogenes: a short overview. Microbes Infect 9: 1208-1215, 2007.

13. Darji A, Mohamed W, Domann E and Chakraborty T: Induction of immune responses by attenuated isogenic mutant strains of Listeria monocytogenes. Vaccine 21 (Suppl 2): 102-109, 2003.

14. Wood LM, Pan ZK, Guirnalda P, Tsai P, Seavey M and Paterson Y: Targeting tumor vasculature with novel Listeriabased vaccines directed against CD105. Cancer Immunol Immunother 60: 931-942, 2011.

15. Wallecha A, Maciag PC, Rivera S, Paterson Y and Shahabi V: Construction and characterization of an attenuated Listeria monocytogenes strain for clinical use in cancer immunotherapy. Clin Vaccine Immunol 16: 96-103, 2009.

16. Seavey MM, Pan ZK, Maciag PC, et al: A novel human Her-2/ neu chimeric molecule expressed by Listeria monocytogenes can elicit potent HLA-A2 restricted CD8-positive T cell responses and impact the growth and spread of Her-2/neu-positive breast tumors. Clin Cancer Res 15: 924-932, 2009.

17. Maciag PC, Radulovic S and Rothman J: The first clinical use of a live-attenuated Listeria monocytogenes vaccine: A Phase I safety study of Lm-LLO-E7 in patients with advanced carcinoma of the cervix. Vaccine 27: 3975-3983, 2009.

18. Sewell DA, Pan ZK and Paterson Y: Listeria-based HPV-16 E7 vaccines limit autochthonous tumor growth in a transgenic mouse model for HPV-16 transformed tumors. Vaccine 26 : 5315-5320, 2008.

19. Gunn GR, Zubair A, Peters C, Pan ZK, Wu TC and Paterson Y: Two Listeria monocytogenes vaccine vectors that express different molecular forms of human papilloma virus-16 (HPV-16) E7 induce qualitatively different $\mathrm{T}$ cell immunity that correlates with their ability to induce regression of established tumors immortalized by HPV-16. J Immunol 167: 6471-6479, 2001.

20. Wallecha A, French C, Petit R, Singh R, Amin A and Rothman J: Lm-LLO-based immunotherapies and HPV-associated disease. J Oncol 2012: 542851, 2012.

21. Verch T, Pan ZK and Paterson Y: Listeria monocytogenesbased antibiotic resistance gene-free antigen delivery system applicable to other bacterial vectors and DNA vaccines. Infect Immun 72: 6418-6425, 2004

22. Cossart P and Toledo-Arana A: Listeria monocytogenes, a unique model in infection biology: an overview. Microbes Infect 10: 1041-1050, 2008.

23. Pamer EG: Immune responses to Listeria monocytogenes. Nat Rev Immunol 4: 812-823, 2004.
24. Schlüter D, Domann E, Buck C, et al: Phosphatidylcholinespecific phospholipase $\mathrm{C}$ from Listeria monocytogenes is an important virulence factor in murine cerebral listeriosis. Infect Immun 66: 5930-5938, 1998

25. Angelakopoulos H, Loock K, Sisul DM, Jensen ER, Miller JF and Hohmann EL: Safety and shedding of an attenuated strain of Listeria monocytogenes with a deletion of act $\mathrm{A} / \mathrm{plcB}$ in adult volunteers: a dose escalation study of oral inoculation. Infect Immun 70: 3592-3601, 2002.

26. Brockstedt DG and Dubensky TW: Promises and challenges for the development of Listeria monocytogenes-based immunotherapies. Expert Rev Vaccines 7: 1069-1084, 2008.

27. Monk IR, Gahan CG and Hill C: Tools for functional postgenomic analysis of Listeria monocytogenes. Appl Environ Microbiol 74: 3921-3934, 2008.

28. Yin Y, Tian D, Jiao H, et al: Pathogenicity and immunogenicity of a mutant strain of Listeria monocytogenes in the chicken infection model. Clin Vaccine Immunol 18: 500-505, 2011.

29. Jiao X, Lo-Man R, Winter N, Dériaud E, Gicquel B and Leclerc C: The shift of Th1 to Th2 immunodominance associated with the chronicity of Mycobacterium bovis bacille Calmette- Guérin infection does not affect the memory response. J Immunol 170: 1392-1398, 2003.

30. Berraondo P, Nouzé C, Préville X, Ladant D and Leclerc C: Eradication of large tumors in mice by a tritherapy targeting the innate, adaptive, and regulatory components of the immune system. Cancer Res 67: 8847-8855, 2007.

31. Naito S, von Eschenbach AC, Giavazzi R and Fidler IJ: Growth and metastasis of tumor cells isolated from a human renal cell carcinoma implanted into different organs of nude mice. Cancer Res 46: 4109-4115, 1986.

32. Lin K, Doolan K, Hung CF and Wu TC: Perspectives for preventive and therapeutic HPV vaccines. J Formos Med Assoc 109: 4-24, 2010.

33. Yin YL, Zhang CJ, Tian DB, Pan ZM and Jiao XA: Kinetics of mutant Listeria monocytogenes strain yzuLM1-2 in murine infection model. Vet Sci in China 37: 850-853, 2007.

34. de Jong A, van der Hulst JM, Kenter GG, et al: Rapid enrichment of human papillomavirus (HPV)-specific polyclonal T cell populations for adoptive immunotherapy of cervical cancer. Int J Cancer 114: 274-282, 2005.

35. Vesely MD, Kershaw MH, Schreiber RD and Smyth MJ: Natural innate and adaptive immunity to cancer. Annu Rev Immunol 29: 235-271, 2011.

36. Yu YA, Shabahang S, Timiryasova TM, et al: Visualization of tumors and metastases in live animals with bacteria and vaccinia virus encoding light-emitting proteins. Nat Biotechnol 22: 313-320, 2004

37. Gravekamp C and Paterson Y: Harnessing Listeria monocytogenes to target tumors. Cancer Biol Ther 9: 257-265, 2010.

38. Zeng Q, Peng S, Monie A, et al: Control of cervicovaginal HPV-16 E7-expressing tumors by the combination of therapeutic HPV vaccination and vascular disrupting agents. Hum Gene Ther 22: 809-819, 2010.

39. Sharma RK, Schabowsky RH, Srivastava AK, et al: 4-1BB ligand as an effective multifunctional immunomodulator and antigen delivery vehicle for the development of therapeutic cancer vaccines. Cancer Res 70: 3945-3954, 2010.

40. Ostrand-Rosenberg S: Animal models of tumor immunity, immunotherapy and cancer vaccines. Curr Opin Immunol 16: $143-150,2004$. 